\title{
Facilitadores e barreiras para o compartilhamento de conhecimento em cadeias de suprimentos
}

\author{
Felipe Nodari \\ Programa de Pós-Graduação em Administração \\ Pontifícia Universidade Católica do Rio Grande do Sul-PUC-RS \\ fnodari@gmail.com \\ Eduardo Kunzel Teixeira \\ Programa de Pós-Graduação em Administração \\ Pontifícia Universidade Católica do Rio Grande do Sul-PUC-RS \\ eduardo.kunzel@hotmail.com

\section{Mirian Oliveira} \\ Programa de Pós-Graduação em Administração \\ Pontifícia Universidade Católica do Rio Grande do Sul-PUC-RS \\ miriano@pucrs.br
}

\author{
Aurora Carneiro Zen \\ Programa de Pós-Graduação em Administração \\ Universidade Federal do Rio Grande do Sul-UFRGS \\ aczen@ea.ufrgs.br
}

\section{RESUMO}

O compartilhamento do conhecimento constitui um processo que influencia o sucesso organizacional. Este trabalho analisou os fatores que facilitam ou dificultam o compartilhamento do conhecimento em um contexto de cadeia de suprimentos. Para tanto, foi desenvolvido um estudo qualitativo do tipo exploratório, onde agentes da indústria de máquinas e equipamentos do estado do Rio Grande do Sul foram entrevistados a respeito da percepção das barreiras e facilitadores para o compartilhamento de conhecimento em sua cadeia. Como resultado, obteve-se um conjunto de facilitadores e outro de barreiras ao compartilhamento de conhecimento, assim como a relação entre eles.

Palavras-chave: Compartilhamento do conhecimento, Facilitadores, Barreiras, Cadeia de suprimentos.

\begin{abstract}
Knowledge sharing is a process that influences organizational success. This study analyzed factors that facilitate or hinder knowledge sharing in a context of supply chain. For this purpose, we developed an exploratory qualitative study, where agents of machinery and equipment industry in the state of Rio Grande do Sul were interviewed about the perception of barriers and facilitators to knowledge sharing in their chain. As a result, a set of facilitators and other of barriers to knowledge sharing were obtained, as well as the relationship between them.
\end{abstract}

Keywords: Knowledge sharing, Facilitators, Barriers, Supply chain.

\section{Introdução}

Atualmente, o conhecimento é identificado como um dos ativos mais importantes (SIAKAS; GEORGIADOU; BALSTRUP, 2010), e um relevante fator para a viabilidade de uma organização (FRIESL; SACKMANN; KREMSER, 2011). Representa, também, uma fonte de vantagem efetiva caso o conhecimento individual e de grupos seja convertido em conhecimento organizacional (KARKOULIAN; AL HARAKE; MESSARRA, 2010; KHAN, 2010).

A gestão do conhecimento tem sido reconhecida como o ponto de mudança entre a economia dos recursos naturais e do capital intelectual (TENG; SONG, 2011), e o compartilhamento de conhecimento é considerado importante processo na gestão do conhecimento, possibilitando aumento de desempenho para a organização 
(VELMURUGAN; KOGILAH; DEVINAGA, 2010). Desta forma, o compartilhamento de conhecimento constitui um processo essencial para o sucesso organizacional, como melhora no tempo de resposta, produtividade, aprendizado e capacidade de inovação (KARKOULIAN; AL HARAKE; MESSARRA, 2010), podendo ocorrer dentro das empresas ou entre elas.

A prática do compartilhamento de conhecimento nas cadeias de suprimentos tem se tornado comum porque gera vantagem competitiva na cadeia como um todo (ESPALLARDO; OREJUELA; PÉREZ, 2010). Em um contexto de competição global, vendedores e produtores são pressionados para diminuir o custo de estoques e o tempo de resposta. Essa necessidade faz com que o compartilhamento de conhecimento entre as empresas da cadeia de suprimentos tenha importância aumentada (ESPALLARDO; OREJUELA; PÉREZ, 2010). Apesar da importância do processo de compartilhamento de conhecimento, os fatores que facilitam ou dificultam o mesmo ainda não foram propriamente identificados (TOHIDINIA; MOSAKHANI, 2010). Com o intuito de contribuir para o preenchimento desta lacuna, este artigo visa analisar as barreiras e os facilitadores para o compartilhamento de conhecimento, utilizando organizações situadas no contexto de cadeias de suprimento.

Este artigo está estruturado da seguinte forma: na próxima seção, é apresentado o conceito e características da gestão da cadeia de suprimentos (SCM); na terceira seção, é revisada a literatura sobre compartilhamento de conhecimento entre as organizações, suas barreiras e facilitadores, e sua inserção em cadeias de suprimento; na quarta seção, são descritos os procedimentos metodológicos adotados; na quinta seção, os dados são apresentados, analisados e discutidos de acordo com as teorias elencadas; por fim, na última seção são apresentadas as conclusões desta pesquisa.

\section{Gestão da Cadeia de Suprimentos}

Gestão da cadeia de suprimentos (SCM - Supply Chain Management) pode ser definida como um conjunto de abordagens usadas para integrar eficientemente os agentes envolvidos em uma cadeia produtiva de forma que o mercado opere corretamente em termos de quantidades, locais, tempo, custo e satisfação (MEHRJERDI, 2009; THOMAS; ESPER; STANK, 2010).

Esta estrutura visa à eficiência na redução dos riscos e dos custos da gestão de materiais, informação, fluxos financeiros (MEHRJERDI, 2009) e aceleração de operações (THOMAS; ESPER; STANK, 2010; MEHRJERDI, 2009; COOK; HEISER; SENGUPTA, 2011), da origem ao consumo final (MEHRJERDI, 2009). Em síntese, é o conjunto de processos necessários para levar a produção ao consumidor final (VANPOUCKE; BOYER; VEREECKE, 2009).

Práticas de SCM como sistemas de informação interorganizacionais, planejamento colaborativo, mecanismos de compartilhamento de riscos e recompensas e o compartilhamento de informações sobre os consumidores ganham importância, pois melhoram o desempenho das empresas e de seus colaboradores conjuntamente (THOMAS; ESPER; STANK, 2010; HAKANSSON e PERSSON, 2004; COOK; HEISER; SENGUPTA, 2011).

Na ultima década a pesquisa sobre SCM tem envolvido perspectivas como o relacionamento de fornecedores, redes e colaboração (COOK; HEISER; SENGUPTA, 2011), abarcando assuntos que envolvem confiança, comprometimento e interdependência entre firmas (THOMAS; ESPER; STANK, 2010). De fato, um dos tópicos pesquisados na literatura sobre gestão da cadeia de suprimentos trata da integração entre empresas (COOPER; LAMBERT; PAGH, 1997; MEHRJERDI, 2009), que sugere que nas relações de cooperação e integração as empresas obtêm resultados superiores aos alcançado individualmente, desenvolvendo estratégias para alcançar benefícios mútuos (CHEN; PAULRAJ, 2004). De acordo com Hakansson e Persson (2004), a integração de SCM pode ocorrer desde níveis mais individuais, em que a empresa se relaciona com o ambiente externo, até níveis de inter-relação de cadeias, umas com as outras, passando pelo relacionamento entre empresas consumidoras e seus fornecedores diretos, e relações das organizações com todos os elos de sua cadeia.

Malhotra, Gosain e El Sawy (2005) consideram que as relações interorganizacionais apresentam potenciais benefícios de longo e de curto prazo. No curto prazo os benefícios estariam mais associados à eficiência operacional (MALHOTRA; GOSAIN; EL SAWY, 2005; WISNER, 2000), enquanto no longo prazo as relações entre empresas resultariam em benefícios de criação de conhecimento.

Essa visão de integração das atividades almejando resultados melhores que os individuais empresta conceitos da visão baseada em recursos (RBV), que pressupõe valor imerso nas rotinas e processos da firma. Nesse sentido, o relacionamento de empresas pode gerar valor à medida que firmas conectadas combinam, trocam ou criam conhecimento e outros recursos (ESPALLARDO; OREJUELA; PÉREZ, 2010).

\section{Compartilhamento de Conhecimento}

O conhecimento é epistemologicamente classificado em duas dimensões: explícito e tácito (NONAKA; 
TAKEUCHI, 1995). O conhecimento explícito inclui fatos e informações que possam ser expressas em números, palavras ou articuladas em forma de dados, enquanto o tácito contém conhecimentos processuais, percepções e intuições (CHOU, 2005), sendo relativamente mais difícil de ser compartilhado do que o explícito, por ser incorporado socialmente e baseado em experiências pessoais (NONAKA; TAKEUCHI, 1995).

O conhecimento, tanto em sua forma tácita quanto explícita, pode ser compartilhado entre indivíduos. O compartilhamento de conhecimento ocorre quando um indivíduo (ou unidade organizacional) é afetado pelo conhecimento ou experiência de outro (ARGOTE; INGRAM, 2000), o que pode resultar na criação conjunta de novos conhecimentos (SIAKAS; GEORGIADOU; BALSTRUP, 2010; HOOFF; DE RIDDER, 2004). É caracterizado pela atividade de transferência entre pessoas, grupos ou organizações (FORD; STAPLES, 2010), que podem ocorrer através de colaborações formais ou de interações cotidianas informais (FRIESL; SACKMANN; KREMSER, 2011). O compartilhamento de conhecimento formal é feito por canais criados especificamente para o propósito de criar e trocar conhecimento, o informal é feito por mecanismos que facilitam, mas que não são criados para se dedicar a essa atividade (KHAN, 2010). Quando um indivíduo fornece parte de seu conhecimento para outro, independente de ser por mecanismos diretos ou indiretos, ele está envolvido em um processo de compartilhamento (SWIFT; BALKIN; MATUSIK, 2010).

O compartilhamento de conhecimento organizacional ocorre quando os membros da organização trocam informações relacionadas às atividades organizacionais (TOHIDINIA; MOSAKHANI, 2010), incluindo a disseminação do conhecimento entre os membros de equipes e também a incorporação dos que são provenientes do ambiente externo (VELMURUGAN; KOGILAH; DEVINAGA, 2010). O processo de compartilhamento envolve uma troca mútua de conhecimentos explícitos ou implícitos, e pode ser dividido entre dois componentes: o do ponto de vista do recebimento e o do ponto de vista de fornecimento ou doação do conhecimento (HOOFF; DE RIDDER, 2004; KARKOULIAN; AL HARAKE; MESSARRA, 2010). O compartilhamento começa quando o conhecimento alheio é solicitado para preencher alguma lacuna. Pode ocorrer, ainda, de forma voluntária, caso não ocorram solicitações (TENG; SONG, 2011). Entretanto, a localização das fontes de conhecimento necessárias e a transferência deste para a unidade em que será utilizado ou a pessoa que o necessita constituem um processo difícil (DAVENPORT; DE LONG; BEERS, 1998). Neste sentido, a identificação das barreiras ou facilitadores para o compartilhamento de conhecimento representa um importante campo de interesse para as organizações.
Os fatores que possuem influência no compartilhamento de conhecimento, atuando como facilitadores ou barreiras, podem ser classificados em três categorias: tecnológicos, individuais e organizacionais (RIEGE, 2005; MANJIT; JAIN; AHMAD, 2011).

\subsection{Fatores Tecnológicos}

Fatores tecnológicos estão associados ao desempenho ou qualidade no canal de comunicação, falta de integração de sistemas, resistência ao uso de sistemas, a falta de treinamento ou de familiaridade no uso de sistemas, e a viabilidade do trabalho em conjunto através de espaços físicos separados (VELMURUGAN; KOGILAH; DEVINAGA, 2010; TOHIDINIA; MOSAKHANI, 2010; FORD; STAPLES, 2010; TENG; SONG, 2011; MANJIT; JAIN; AHMAD, 2011; LI, 2010), conforme apresentado no Quadro 1.

\begin{tabular}{|l|l|}
\hline \multicolumn{1}{|c|}{ Constructos } & \multicolumn{1}{c|}{ Referências } \\
\hline Qualidade no canal de comunicação & Ford e Staples (2010); Li (2010); Man- \\
Integração de sistemas & jit, Jain e Ahmad (2011); Teng e Song \\
Uso de sistemas & (2011); Tohidinia e Mosakhani (2010); \\
Viabilidade do trabalho em conjunto & Velmurugan, Kogilah e Devinage (2010) \\
& \\
\hline
\end{tabular}

Quadro 1 - Fatores tecnológicos associados ao Compartilhamento de Conhecimento

Fonte: os autores

\subsection{Fatores Individuais}

Os fatores individuais são relativos ao comportamento, percepções e ações individuais e de grupo, dentro ou entre organizações (RIEGE, 2005). Enquanto barreiras para o compartilhamento de conhecimento podem ser representadas pela falta de habilidade de comunicação, reduzida capacidade de absorção do destinatário, ambiguidades casuais sobre o conhecimento e relações árduas entre o emissor e o receptor (MANJIT; JAIN; AHMAD, 2011; LILLEOERE; HANSEN, 2011), a proximidade física, a memória transacional e o relacionamento recíproco podem ser elencados como possíveis facilitadores deste processo associados à comunicação (MANJIT; JAIN; AHMAD, 2011; LILLEOERE; HANSEN, 2011).

A confiança, por sua vez, também é elencada como um importante fator de influência no compartilhamento de conhecimento. Este processo é facilitado em relações de confiança, que pode ser melhorada através de interação social. Relações que apresentam um grau elevado de cuidado pelo outro como, por exemplo, confiança mútua, empatia ativa, acesso à ajuda, leniência em juízo, e coragem, atuam como facilitadores da partilha de conhecimentos (MANJIT; JAIN; AHMAD, 2011; KHAN, 2010; SIAKAS; GEORGIADOU; BALSTRUP, 2010; VELMURUGAN; KOGILAH; DEVINAGA, 2010; LILLEOERE; HANSEN, 2011). 
A percepção limitada ou inexistente dos benefícios pode estar associada com uma atitude hesitante em compartilhar conhecimento, através da percepção de possíveis riscos provenientes deste processo, que podem incluir fatores associados ao reconhecimento pessoal e, a nível organizacional, o acesso a informações estratégicas pelos concorrentes (MANJIT; JAIN; AHMAD, 2011; FRIESL; SACKMANN; KREMSER, 2011).

Um importante fator elencado como facilitador para o processo de compartilhamento de conhecimento é composto pela rede de relacionamentos sociais dos indivíduos. Os laços formais e informais estabelecidos podem influenciar na forma com que o conhecimento pode ser obtido (FRIESL; SACKMANN; KREMSER, 2011; EL HARBI; ANDERSON; AMAMOU, 2011; LILLEOERE; HANSEN, 2011; MANJIT; JAIN; AHMAD, 2011; VELMURUGAN; KOGILAH; DEVINAGA, 2010).

Diferentes experiências ou diferentes nacionalidades levam a diferentes expectativas, que podem representar barreiras para o processo de compartilhamento de conhecimento. Normas subjetivas, recompensas extrínsecas, confiança social, senso de auto-estima através do comportamento de compartilhamento de conhecimentos são elencadas como potenciais facilitadores deste processo (MANJIT; JAIN; AHMAD, 2011; SIAKAS; GEORGIADOU; BALSTRUP, 2010; TOHIDINIA; MOSAKHANI, 2010; VELMURUGAN; KOGILAH; DEVINAGA, 2010; DONG; CHAU; GROSSMAN, 2010). O distanciamento do poder, individualismo/coletivismo, machismo, aversão a incerteza e a orientação ao longo prazo, pertencentes a algumas culturas, podem determinar a importância e a utilidade do aprendizado a partir do compartilhamento informal de conhecimento (KHAN, 2010). O compartilhamento do conhecimento pode depender de ações voluntárias de abertura, cuidado e atenção, confiança e tolerância frente a diferenças culturais. Esses valores são em parte culturais, mas não necessariamente antecedentes da cultura (SIAKAS; GEORGIADOU; BALSTRUP, 2010). Os fatores individuais e os respectivos autores pesquisados estão relacionados no Quadro 2.

\begin{tabular}{|c|c|}
\hline Constructos & Referências \\
\hline Habilidade de comunicação & $\begin{array}{l}\text { Lilleoere e Hansen (2011); Manjit, Jain e Ahmad } \\
\text { (2011) }\end{array}$ \\
\hline Confiança & $\begin{array}{l}\text { Khan (2010); Lilleoere e Hansen (2011); Manjit, } \\
\text { Jain e Ahmad (2011); Siakas, Georgiadou e Bals- } \\
\text { trup (2010); Velmurugan, Kogilah e Devinaga } \\
(2010)\end{array}$ \\
\hline $\begin{array}{l}\text { Rede de Relacionamentos } \\
\text { Sociais }\end{array}$ & $\begin{array}{l}\text { El Harbi, Anderson e Amamou (2011); Friesl, } \\
\text { Sackmann e Kremser (2011); Lilleoere e Hansen, } \\
\text { (2011); Manjit, Jain e Ahmad (2011); Velmurugan, } \\
\text { Kogilah e Devinaga (2010) }\end{array}$ \\
\hline Diferenças Culturais & $\begin{array}{l}\text { Dong, Chau e Grossman (2010); Manjit, Jain e } \\
\text { Ahmad (2011); Siakas, Georgiadou e Balstrup } \\
\text { (2010); Tohidinia e Mosakhani (2010); Velmuru- } \\
\text { gan, Kogilah e Devinaga (2010) }\end{array}$ \\
\hline
\end{tabular}

\begin{tabular}{|c|l|} 
(... continuação) \\
\hline Recompensas Percebidas & $\begin{array}{l}\text { Friesl, Sackmann e Kremser (2011); Manjit, Jain } \\
\text { e Ahmad (2011) }\end{array}$ \\
\hline Percepção de risco & $\begin{array}{l}\text { Friesl, Sackmann e Kremser (2011); Manjit, Jain } \\
\text { e Ahmad (2011) }\end{array}$ \\
\hline
\end{tabular}

Quadro 2 - Fatores individuais associados ao Compartilhamento de Conhecimento

Fonte: os autores

\subsection{Fatores Organizacionais}

Os fatores organizacionais, relativos ao ambiente corporativo e ao contexto organizacional, em convergência com os fatores individuais, influenciam o processo de compartilhamento de conhecimento (RIEGE, 2005). Empresas com culturas orgânicas, caracterizadas por ambientes de trabalho dinâmicos, empreendedores e criativos, possuem maior facilidade na implantação de processos de compartilhamento de conhecimento, o que não é observado em organizações com foco em obter vantagens competitivas através das transações (SUPPIAH; MANJIT, 2011; SIAKAS; GEORGIADOU; BALSTRUP, 2010)

Existem controvérsias a respeito da influência de estruturas hierárquica verticais no efeito negativo no compartilhamento de conhecimento. Entretanto, estruturas baseadas em equipes e coordenação horizontal facilitam o compartilhamento (FRIESL; SACKMANN; KREMSER, 2011; SUPPIAH; MANJIT, 2011; MANJIT; JAIN; AHMAD, 2011).

A falta de uma cultura de compartilhamento de conhecimento, onde relações de poder são constituídas através do acúmulo de conhecimento possuem denotada relação com a falta de motivação para o compartilhamento de conhecimento (MANJIT; JAIN; AHMAD, 2011; LILLEOERE; HANSEN, 2011). Por outro lado, a existência de incentivos para o compartilhamento, através de um sistema de recompensa explícito para o indivíduo, é positivamente relacionada com o compartilhamento de conhecimento (MANJIT; JAIN; AHMAD, 2011; TOHIDINIA; MOSAKHANI, 2010; LILLEOERE; HANSEN, 2011).

O incentivo ao compartilhamento também pode ser desenvolvido pela organização através do desenvolvimento de uma identidade comum entre os indivíduos envolvidos neste processo. Na medida em que os funcionários sabem a localização dos recursos de conhecimento, o trabalho de equipe, programas para envolvimento do funcionário, e alto envolvimento entre empresa e funcionário podem potencializar os benefícios associados ao compartilhamento de conhecimento (LAURING; SELMER, 2011; TOHIDINIA; MOSAKHANI, 2010; KARKOULIAN; AL HARAKE; MESSARRA, 2010; 
SUPPIAH; MANJIT, 2011; LILLEOERE; HANSEN, 2011). A cultura nacional influencia fortemente a partilha do conhecimento, mas a cultura organizacional também está relacionada. A cultura nacional é considerada menos maleável que a cultura organizacional, mas se a cultura organizacional é muito forte, pode ser observada a influência desta sobre o compartilhamento do conhecimento. Nesse sentido, empresas multinacionais fortalecem sua cultura organizacional para tentar controlar os efeitos da cultura nacional (SIAKAS; GEORGIADOU; BALSTRUP, 2010). Os gestores possuem um papel essencial garantindo programas de recompensas, facilitando o acesso a tecnologias e na auditoria das atividades (TOHIDINIA; MOSAKHANI, 2010), mas, por outro lado, são essencialmente os funcionários que decidem trocar conhecimento ou não. Nesse sentido, motivação e conhecimento pregresso são essenciais.

Os fatores organizacionais que podem ser relacionados como barreiras e facilitadores para o compartilhamento de conhecimento estão relacionados a seguir, no Quadro 3.

\begin{tabular}{|c|l|}
\hline $\begin{array}{c}\text { Constructos } \\
\begin{array}{c}\text { Cultura de } \\
\text { compartilhamento }\end{array}\end{array}$ & $\begin{array}{l}\text { Lilleoere e Hansen (2011); Manjit, Jain e Ahmad } \\
\text { (2011); Suppiah e Manjit (2011); Siakas, Georgia- } \\
\text { doue Balstrun (2010) }\end{array}$ \\
\hline Estrutura Organizacional & $\begin{array}{l}\text { Friesl, Sackmann e Kremser (2011); Manjit, Jain e } \\
\text { Ahmad (2011); Suppiah e Manjit (2011) }\end{array}$ \\
\hline Alta Administração & Tohidinia e Mosakhani (2010) \\
\hline $\begin{array}{c}\text { Incentivos ao } \\
\text { compartilhamento }\end{array}$ & $\begin{array}{l}\text { Manjit, Jain e Ahmad (2011); Tohidinia e Mo- } \\
\text { sakhani (2010); Lilleoere e Hansen (2011) }\end{array}$ \\
\hline Identidade comum & $\begin{array}{l}\text { Lauring e Selmer (2011); Tohidinia e Mosakhani } \\
\text { (2010); Karkoulian, Al Harake e Messarra (2010); } \\
\text { Suppiah e Manjit (2011); Lilleoere e Hansen } \\
\text { (2011) }\end{array}$ \\
\hline Conhecimento pregresso & Tohidinia e Mosakhani (2010) \\
\hline
\end{tabular}

Quadro 3 - Fatores organizacionais associados ao Compartilhamento de Conhecimento

Fonte: os autores

De acordo com a revisão da literatura apresentada, os constructos identificados como possíveis barreiras ou facilitadores para o processo de compartilhamento de conhecimento serão explorados dentro do contexto da gestão de cadeias de suprimento, de acordo com o direcionamento metodológico descrito a seguir.

\section{Método de Pesquisa}

Este é um estudo qualitativo da percepção dos agentes envolvidos em uma cadeia de suprimentos sobre o compartilhamento de conhecimento interorganizacional, e caracteriza-se como exploratório, visando aumentar o conhecimento sobre o tema (MALHOTRA, 2001).

As empresas selecionadas pertencem à indústria de máquinas e equipamentos do estado do Rio Grande do
Sul, no Brasil. O critério de seleção dos setores investigados foi o da relevância para a economia do referido estado. De acordo com a Fundação de Economia e Estatística (FEE-RS), entre 2009 e 2010 o valor agregado bruto (VAB) da indústria foi o que mais cresceu $(10,3 \%)$ neste estado brasileiro, impulsionado pela indústria de transformação, cujos destaques foram os setores mencionados. No referido período, o da indústria de máquinas e equipamentos cresceu 27,5\%. As empresas foram selecionadas por conveniência.

As empresas pertencem a duas cadeias distintas, nos setores de siderurgia e de ferramentas motorizadas, incluído as duas empresas líderes da cadeia, ambas multinacionais de grande porte, consideradas referência em seus mercados de atuação. As demais empresas pesquisadas possuem até 99 funcionários e faturamento inferior a $\mathrm{R} \$ 2.400 .000$, compreendendo as atividades de automação industrial, implementos rodoviários e desenvolvimento e comércio de materiais de fixação e abrasivos. A Figura 1 apresenta a descrição resumida das empresas pesquisadas, incluindo seus setores de atuação e a estrutura dos relacionamentos.

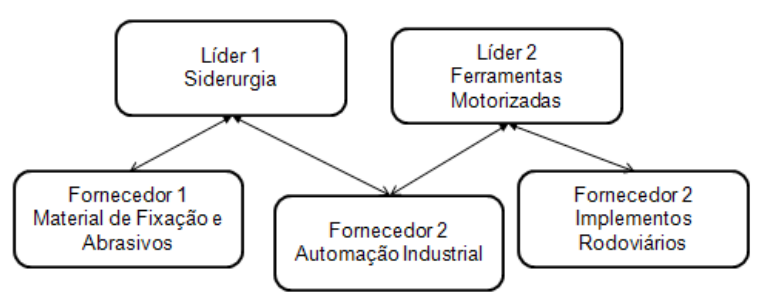

Figura 1 -Relacionamento entre as empresas pesquisadas

As dimensões reveladas na revisão da literatura foram convertidas em questões para um roteiro de entrevista semiestruturada, de acordo com Flick (2009). Estas questões foram avaliadas e validadas por um pesquisador, com experiência na área do estudo.

As entrevistas foram conduzidas com o auxílio de tecnologias de comunicação remota - gravadas com o consentimento dos entrevistados - cuja duração média foi de trinta minutos.

Cada entrevista foi transcrita por um pesquisador que cuja língua materna é a mesma do entrevistado. As entrevistas referentes às empresas fornecedoras das empresas foram realizadas com os diretores e/ou proprietários das mesmas. Para as empresas líderes de cadeia, foi entrevistado o gerente da área diretamente responsável pelo relacionamento com os fornecedores, representada, nas empresas entrevistadas, pela gerência de suprimentos ou de compras.

As respostas foram analisadas e categorizadas por codificação temática, utilizando como base teórica de 
codificação o próprio quadro de constructos desenvolvido na revisão da literatura.

A codificação foi feita com o auxílio do software MAXQDA $^{\circledR}$ seguindo os passos:

Etapa 1 - Codificação por um pesquisador, utilizando a codificação temática (FLICK, 2009; GIBBS, 2009) a partir da revisão da literatura revelou;

Etapa 2 - Entrega das entrevistas e do livro de códigos para o segundo pesquisador, que fez o mesmo processo de codificação realizado pelo primeiro pesquisador. As atividades foram realizadas independentemente;

Etapa 3 - Comparação dos casos e discussão dos pontos discordantes.

Como resultado das codificações independentes, os pesquisadores obtiveram $80 \%$ de concordância. Os $20 \%$ restantes foram discutidos até o alcance do consenso. O objetivo das etapas 2 e 3 (processo de triangulação) é aumentar a validade da pesquisa através da comparação de codificações independentes que consideram os mesmos critérios. Essa técnica possibilita esclarecer interpretações divergentes e também revelar elementos que não identificados em análise única (FLICK, 2009; GIBBS, 2009).

\section{Análise e Discussão dos Resultados}

Nesta seção é apresentada a análise do conteúdo das entrevistas, avaliando sua relação e adequação com os temas e tópicos sugeridos pela revisão da literatura.

\subsection{Fatores Tecnológicos}

Houve concordância dos entrevistados sobre a influência direta que a tecnologia tem no compartilhamento de conhecimento, ajudando nas atividades diárias de comunicação, sentido tanto na transmissão quanto na recepção do conhecimento. Dentre as ferramentas utilizadas, os entrevistados mencionaram basicamente ferramentas de comunicação instantânea. A dependência da tecnologia para o cumprimento das atividades, resultado este compatível com os apresentados por Velmurugan, Kogilah e Devinaga (2010).

Dentre os problemas relacionados à tecnologia e seu uso para o compartilhamento do conhecimento os entrevistados destacaram a falta de aptidão e de conhecimentos prévios da mão de obra em lidar com as tecnologias. As barreiras relacionadas ao acesso à tecnologia, incluindo a aptidão para seu uso, são compatíveis com os resultados apresentados por Manjit, Jain e Ahmad (2011). O entrevistado Líder 1, por sua vez, também destaca que as diferenças culturais podem atuar como redutores da eficiência e agilidade da tecnologia, sendo este um fator em que sua influência no processo de compartilhamento pode ser melhor explorada.

Tohidinia e Mosakhani (2010) e Teng e Song (2011) apresentam que o ambiente para compartilhamento de conhecimento é positivamente afetado pelo nível de tecnologias de informação e comunicação utilizadas, resultados estes compatíveis com as opiniões dos entrevistados desta pesquisa.

\subsection{Fatores Individuais}

A dificuldade em estabelecer a comunicação entre as empresas ficou evidente pelas respostas de alguns entrevistados, inclusive sendo considerada pelo entrevistado Líder 2 como uma das principais barreiras ao compartilhamento de conhecimento. Esta importância também é apresentada por Manjit, Jain e Ahmad (2011).

Os entrevistados atribuíram importância ao conhecimento pregresso dos agentes na hora de fazer uma comunicação efetiva, como pode ser ilustrado pela fala do entrevistado Fornecedor 2: “[...] a pessoa que não tem tanto didática, ou não tem desenvoltura para repassar o conhecimento, acaba impactando na transferência desse conhecimento.". Por outro lado, sai fortalecida a importância do conhecimento tácito no compartilhamento de conhecimento, dado que a proximidade física e o contato continuado foram mencionados como formas de resolver impasses na comunicação entre empresas, o que é compatível com os resultados apresentados por Lilleoere e Hansen (2011).

O entrevistado Líder 1 foi o único que mencionou dificuldades na comunicação oriundas de diferenças culturais entre os funcionários das empresas. Sobressai aqui a ideia de choque cultural advinda dos costumes, mas também o da diferença etária.

Destaca-se o ponto relacionado pelo entrevistado Fornecedor 1, que salientou os efeitos nocivos à comunicação oriunda das normatizações que regem o comportamento das empresas. Segundo este entrevistado, tais barreiras legais ao raio de ação conferem confusão ao processo de comunicação, criando problemas.

A importância de conhecer e se relacionar com as outras empresas, criando a memória transacional e a relação de interdependência, são questões que ficam explícitas nas considerações do entrevistado Fornecedor 3, quando diz que a diferença entre conhecimentos é a principal barreira ao compartilhamento. 
Também enaltecendo a importância da sinergia entre agentes e a necessidade da memória organizacional, o entrevistado Líder 1 vê a necessidade do fortalecimento de todos os elos da cadeia: "[...] se o fornecedor vai mal, ele nos passa coisas ruins também [...] A gente tem que efetivamente tornar toda a cadeia fortalecida.". Essa posição é reafirmada quando o mesmo entrevistado faz menção ao modelo de gestão utilizada pela empresa Toyota em sua cadeia de fornecedores, um exemplo clássico da literatura de compartilhamento de conhecimento e criação de memória transacional (NONAKA; TAKEUCHI, 1995).

Confiança é um tema central do compartilhamento de conhecimento na percepção de 3 entrevistados. O entrevistado Líder 1, por exemplo, opina ser a falta de laços de confiança a grande barreira ao compartilhamento. De certa forma, essa posição confirma as considerações da literatura de gestão do conhecimento (KHAN, 2010; SIAKAS; GEORGIADOU; BALSTRUP, 2010; VELMURUGAN; KOGILAH; DEVINAGA, 2010; LILLEOERE; HANSEN, 2011), também para a realidade da cadeia de suprimentos. Adicionalmente, a ligação entre conhecimento tácito, contato continuado e confiança também é confirmada com passagens das entrevistas. Como no relato do entrevistado Fornecedor 1: "Eu acho isso bem relevante também, porque tu acaba falando coisas que são mais fechadas para empresas e pessoas que tu tem mais confiança mais contato. " ou do entrevistado Fornecedor 2, ao mencionar que o mecanismo que confere confiança é o contato físico, a proximidade. Existe o entendimento de que os resultados do compartilhamento estão atrelados a existência de uma relação de confiança. A consideração do entrevistado Fornecedor 2 de que "Primeiro vem a confiança, depois vem o resultado." sugere a confiança como um antecedente do compartilhamento em um sentido duplo: sua presença é pré-requisito e também moderadora dos resultados do compartilhamento.

Segundo Das e Teng (2001), relações baseadas em confiança sugerem uma necessidade menor de amparos e mecanismos legais ou formais, o que não encontra confirmação na entrevista do Líder 2. O último ponto destacável em termos de confiança é a percepção de sua maior utilidade quando se transmite o conhecimento do que quando se recebe conhecimento, o que também pode foi observado por Siakas, Georgiadou e Balstrup (2010).

Os aspectos motivadores ou que influenciam a intenção de compartilhar conhecimento ficaram centrados em dois pontos: dois entrevistados (Fornecedor 1 e Fornecedor 2) tiveram a percepção de que a condição de acessar o conhecimento alheio e poder aprender com suas experiências e capacidades influencia fortemente a intenção de compartilhar conhecimento; e outro entrevistado (Líder 2) vê no fator econômico a motivação essencial para que o compartilhamento ocorra, o que também foi observado por Tohidinia e Mosakhani (2010).

A rede de relacionamentos foi constatada como impactante sobre o compartilhamento de conhecimento. Líder 2, Fornecedor 2, Fornecedor 1, Líder 1 e Fornecedor 3 consideraram que os laços sociais entre os elementos da rede, isto é, as pessoas manterem contatos não estritamente corporativos, como positivamente impactantes sobre o compartilhamento. Por outro lado, dois pontos merecem destaque: Líder 1 enfatiza tanto a qualidade dos laços como a quantidade de laços e Fornecedor 3 considera que à medida que o conhecimento se torna mais técnico, mais específico, o efeito dos laços sociais sobre o compartilhamento vão ficando menores. A qualidade dos laços citadas pelo Líder 1 pode ser relacionada com o observado por El Harbi, Anderson e Amamou (2011), que a reciprocidade de vantagens para as redes de relacionamento com parceiros são fundadas sobre bem-estabelecidas relações de confiança.

As entrevistas mantiveram as constatações teóricas sobre a influência negativa das culturas nacionais sobre o compartilhamento de conhecimento (VELMURUGAN; KOGILAH; DEVINAGA, 2010; SIAKAS; GEORGIADOU; BALSTRUP, 2010; MANJIT; JAIN; AHMAD, 2011), tanto que o Líder 2 considera que a cultura é a principal barreira ao compartilhamento. Nessa mesma linha, Líder 1 vê na força da cultura organizacional, do comprometimento dos gestores e da convergência de interesses a capacidade de sufocar a resistência ou incompatibilidades que a cultura nacional possa oferecer. Diferentes valores, necessidades e fatores motivacionais apresentados em diferentes culturas (VELMURUGAN; KOGILAH; DEVINAGA, 2010) também foram observados pelos entrevistados Líder 1 e Líder 2, como uma importante barreira ao compartilhamento.

Adicionalmente, foram encontradas considerações sobre burocracia e sua relação com a cultura. Quando indagado sobre a influência que a cultura possa ter no compartilhamento de conhecimento, o entrevistado Fornecedor 2 mencionou uma experiência com uma empresa europeia, e que esta relação seria muito mais ágil. Em sua resposta ele atribui à cultura dos países a responsabilidade pela burocratização das relações. " [...] a velocidade de execução de uma ação é mais rápida com a cultura europeia, é mais decidido, no Brasil tu depende de leis e outras coisas" (Fornecedor 2).

Entre os entrevistados, os principais itens mencionados foram o crescimento e evolução da empresa, o ganho econômico, a redução de custos e o aumento da produtividade.

De uma forma geral, eles percebem que acessar o conhecimento alheio conferirá um diferencial para empresa, e 
este diferencial tem efeitos sobre o desenvolvimento e evolução da empresa. Por outro lado, é forte a conexão das recompensas percebidas com o retorno financeiro. Reduzir custos melhorar a produtividade são questões mais pontuais dentro dos benefícios que trarão evolução da organização.

Um último ponto a destacar sobre as recompensas percebidas é que não foi detectada uma intenção de compartilhar conhecimento se a empresa não obtiver benefícios vindos desta ação. $\mathrm{O}$ entrevistado Fornecedor 2 demonstra que se em uma ação conjunta o benefício é apenas para a outra empresa, e sua empresa obterá benefícios somente no futuro, a disposição a compartilhar é mais difícil. Cada ação deve vir vinculada com benefícios percebidos diretamente dela. Esta posição diverge da apresentada por Manjit, Jain e Ahmad (2011), que posiciona as barreiras relacionadas à exposição do benefício com o compartilhamento de conhecimento com pouca relevância.

Em geral, a percepção de risco é reduzida nos casos em que o conhecimento é disponibilizado pelo parceiro. Por outro lado, foi admitido que certas informações podem não ser disponibilizadas sem mecanismos formais de proteção. De certa forma, estas considerações são indícios de que o compartilhamento de conhecimento não é realizado pelo contato contínuo e muito próximo, o que significa que as empresas protegem seu conhecimento simplesmente não mostrando documentos, processos e instalações. Se o contato fosse extremamente continuado e próximo, certamente as empresas necessitariam de mecanismos mais robustos de proteção do conhecimento. Essa ausência de proteções formais não é a realidade dos entrevistados Fornecedor 2 e Líder 1. Enquanto o primeiro admite que suas ações de compartilhamento de conhecimento são formalizadas (e menciona que a grande maioria de seus parceiros agem da mesma forma) o segundo mantém formalidades inclusive quanto a ética de seus fornecedores, obrigando-os a assinar um documento de compromisso em manter uma conduta condizente com a cultura organizacional da Líder 1. Em contrapartida, o entrevistado Fornecedor 2 afirma que as formalidades contratuais são uma realidade no mundo empresarial muito rígida, sem substitutos ou flexibilizadores. Mesmo que os contratos não sejam muito restritivos ou detalhados, existe a necessidade de estabelecimento de pactos formais nas ações. Quando questionado sobre a possibilidade da confiança reduzir a necessidade dos contratos, Fornecedor 2 explica que: "[...] pela necessidade, hoje em dia, de formalização, de documentos, [...]. São raros os momentos hoje que a gente pode ter uma troca sem ter alguma coisa formalizada.".

\subsection{Fatores Organizacionais}

As entrevistas apontaram que a cultura de compartilhamento de conhecimento é estimulada internamente nas empresas, mas nem tanto entre empresas. Foram identificados elementos nas respostas que são contrárias ao pensamento de compartilhamento de conhecimento, como a valorização da exclusividade do conhecimento, e não da capacidade de gerar mais conhecimento quando este é partillhado. De fato, a equalização entre conhecimento e poder é mencionada como uma barreira ao compartilhamento do conhecimento por Lilleoere e Hansen (2011) e também por Manjit, Jain e Ahmad (2011).

Os entrevistados não ressaltaram influência positiva do tipo de estrutura hierárquica no compartilhamento de conhecimento. Entretanto, a atividade de compartilhar conhecimento tem seu efeito reduzido em estruturas hierárquicas verticalizadas, onde o agente depende do aval de altos níveis hierárquicos para o compartilhamento. Este resultado é compatível com os trabalhos de Friesl, Sackmann e Kremser (2011) e de Suppiah e Manjit (2011), que ressaltam a estrutura hierárquica como barreira ao compartilhamento de conhecimento.

Outras questões relativas à hierarquia foram observadas em relação ao perfil da administração. Segundo o entrevistado Líder 1: "[...] não é o tamanho da hierarquia, mas o perfil da hierarquia [...]". Embora essa circunstância tenha efeito reduzido sobre o compartilhamento, demonstra que tem efeito importante sobre sua ocorrência ou não.

O perfil do gestor da alta administração da organização é reconhecido como fundamental para a incorporação de práticas associadas ao compartilhamento de conhecimento entre as empresas. Como pode ser observado no seguinte depoimento "[...] eu acho que depende muito da cultura e da cabeça até das pessoas que gerenciam essa empresa, em disseminar o conhecimento ou não [...]” (Fornecedor 3). Uma significativa barreira para o compartilhamento de conhecimento pode ser constituída nos casos em que gestores de níveis hierárquicos superiores não reconheçam as práticas relacionadas com a gestão do conhecimento como de interesse da organização. $\mathrm{O}$ apoio a esses processos pode não garantir a sua implantação, mas a falta de corresponde a um importante limitador para a disseminação dessa prática, com influência, inclusive, no estabelecimento da cultura de compartilhamento de conhecimento na organização.

Foram observadas controvérsias, em relação à influência dos incentivos quanto ao compartilhamento de conhecimento. Por um lado, em uma das empresas líderes da cadeia, o incentivo para o compartilhamento é apontado como uma prática estabelecida, como relatado pelo entrevistado Líder 1: “[...] e esses grupos são remunerados financeiramente por idéias que eles dão no sentido 
de contribuir com a melhoria do processo produtivo [...] essa metodologia fica disponível em um banco de dados, e ela é compartilhada com todas as operações do mundo inteiro, [...] e se eu buscar essa metodologia já existente do banco de dados da gestão do conhecimento, eu também vou ser remunerado e a minha equipe vai ser reconhecida por utilizar uma prática existente [...]". Entretanto, para as outras empresas (Fornecedor 1 e 3), de menor porte, é relatado que, devido a influências culturais, esta prática não demonstra obter os resultados esperados. Em ambos os casos, a influência de fatores culturais é notadamente observada, e, por isso, é sugerido que fatores culturais são moderadores na influência de incentivos e de sistemas de recompensas explícitos no processo de compartilhamento de conhecimento. Essa reduzida relevância das recompensas percebidas é ratificada pelo estudo de Tahidinia e Mosakhani (2010).

A criação de uma identidade comum é observada. De acordo com o entrevistado Líder 1, existe a participação em grupos com essa finalidade, de acordo com seguinte relato: "[...] nós tínhamos um grupo formado por grandes empresas, e com perfil, vamos dizer assim, de aquisição, mais ou menos parecido, e nosso objetivo era disseminar a troca de práticas, e têm vários outros exemplos, na área de segurança, na área de medicina do trabalho, na área de qualidade, na área de meio ambiente [...]". O mesmo posicionamento é observado também pelo agente Fornecedor 3, que enfatiza claramente a influência de uma identidade comum no compartilhamento de conhecimento. Esta prática, entretanto, também foi relacionada com a percepção de riscos associados com a abertura de informações estratégicas. Desta forma, sugere-se que a formação de uma identidade comum está positivamente associada com o compartilhamento de conhecimento, porém, moderada pela percepção dos riscos associados com a abertura das informações estratégicas para as empresas de perfil similar, participantes deste grupo. A consideração da relação positiva entre identidade comum e o compartilhamento do conhecimento também é suportada pelo estudo de Karkoulian, Al Harake e Messarra (2010).

Existem circunstâncias que estão intimamente relacionadas com a criação de uma identidade comum, como a convivência próxima, a relação dos agentes em ambientes informais e fora das relações de trabalho e o convívio social. Segundo os entrevistados da pesquisa produzida por Lilleoere e Hansen (2011), estes itens são identificados como facilitadores do compartilhamento do conhecimento.

Os fatores relacionados à cultura organizacional que possuem influência nas práticas de compartilhamento de conhecimento foram particularmente observados nas empresas líderes das cadeias, onde a disseminação de uma cultura própria para os fornecedores mais próximos pôde ser observada, sendo esta fortemente vinculada à percepção dos resultados esperados com os processos de gestão do conhecimento em geral. Os benefícios associados estão fortemente relacionados com a disseminação da cultura organizacional para os fornecedores.

Também é suportada a ideia de que uma cultura dominante é capaz de facilitar o compartilhamento de conhecimento. Suppiah e Manjit (2011) identificaram que um misto de diferentes culturas em que é evidenciada a dominância de uma delas tem efeitos positivos no compartilhamento do conhecimento.

É observado, entretanto, que os dispositivos associados à disseminação da cultura organizacional para as outras empresas pode não representar um instrumento associado à redução dos riscos percebidos associados ao compartilhamento de conhecimento. A existência de instrumentos formais de proteção do conhecimento é relacionada com a disseminação da cultura organizacional, como pode ser observado no seguinte trecho do entrevistado Líder 1: “[...] uma coisa que é bem forte é o código de ética. Nós temos um código de ética, que ele é conhecido, validado, assinado, todas as pessoas são devidamente treinadas e capacitadas por esse código de ética, e nosso papel como contratantes, é que nossos fornecedores também sigam rigorosamente esse código de ética. ". Desta forma, sugere-se a influência da cultura organizacional na cultura de compartilhamento de conhecimento, com uma forte influência da recompensa percebida com esse processo. Entretanto, sua relação com a confiança e com a redução do risco percebido pode ser relativamente menor, devido à manutenção de instrumentos de proteção formais para a proteção do conhecimento compartilhado entre as organizações.

O conhecimento pregresso é relatado como um importante facilitador para o compartilhamento de conhecimento. Desta forma, a existência de conhecimentos anteriores, associados às práticas de compartilhamento de conhecimento, pode estar positivamente relacionada com esse processo. Em concordância com o exposto, Tahidinia e Mosakhani (2010) identificaram que a crença do indivíduo em que domina as capacidades necessárias para atingir o resultado esperado reforça seu comportamento favorável ao compartilhamento do conhecimento.

\subsection{Facilitadores e Barreiras do Processo de Compartilhamento}

Os facilitadores do processo de compartilhamento, no contexto da gestão da cadeia de suprimentos, bem como os possíveis fatores moderadores, de acordo com os entrevistados, estão representados na Figura 2. As relações entre as variáveis pesquisadas com o processo de compartilhamento de conhecimento estão representadas pelas arestas, com a citação dos entrevistados 
que apontaram o item como relevante, e também se o impacto evidenciado é positivo ou negativo. As relações de moderação entre as variáveis são apresentadas pelas arestas que apontam para a relação das demais variáveis com o processo de compartilhamento de conhecimento.

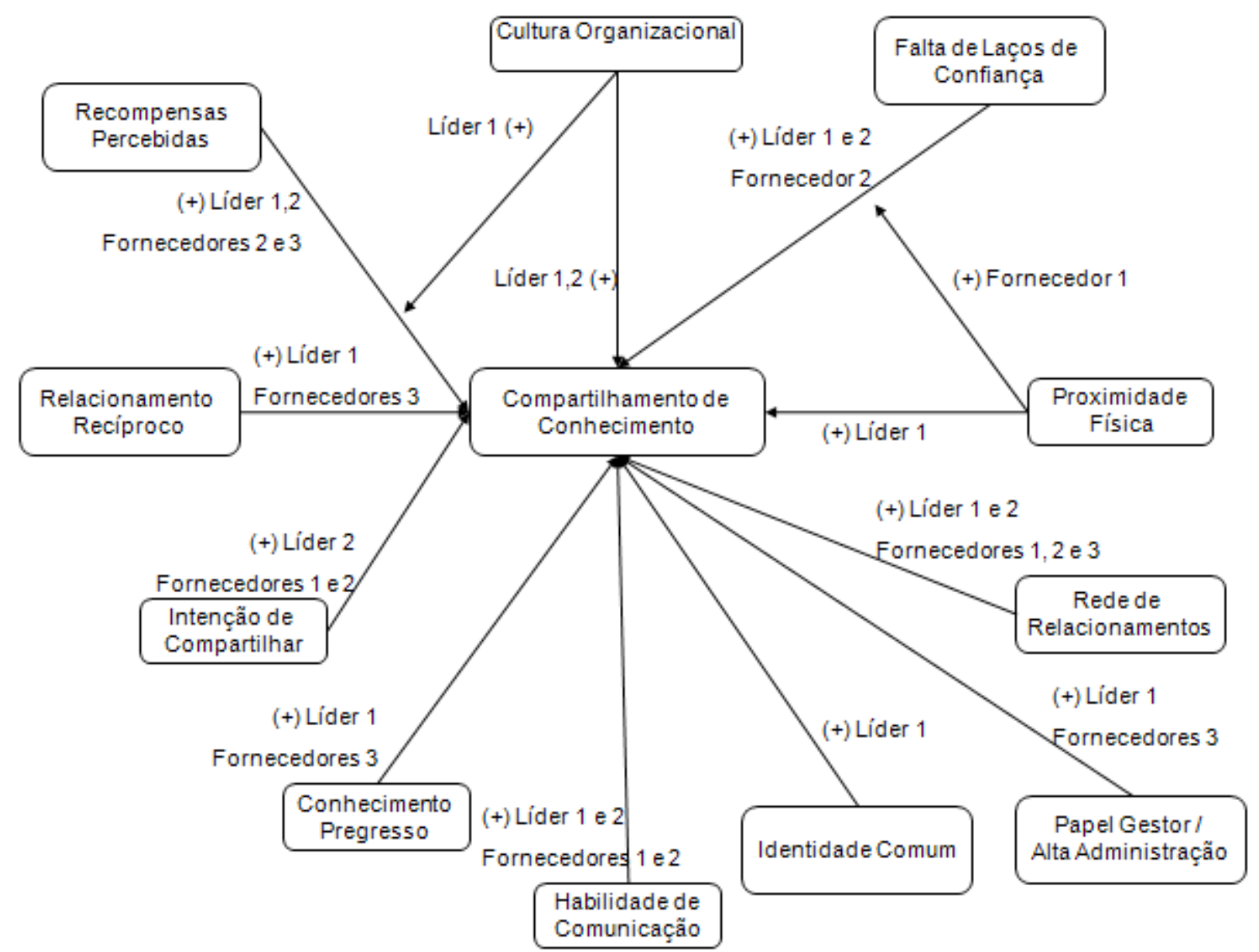

Figura 2 - Facilitadores do processo de compartilhamento de conhecimento em cadeias de suprimento

Fonte: os autores

Conforme demonstrado na Figura 2, para todos os entrevistados a rede de relacionamentos mostra-se como um facilitador do processo de gestão do conhecimento. A habilidade de comunicação e as recompensas percebidas foram apontadas por quatro dos cinco entrevistados, sendo esta última moderada pela cultura organizacional, de acordo com a percepção de uma das empresas líderes de cadeia. O conhecimento pregresso, o relacionamento recíproco, a cultura organizacional, o papel da alta administração foram relacionados, cada um, por dois entrevistados, enquanto a intenção de compartilhar e a criação de laços de confiança foram citadas por três entrevistados cada. A criação de uma identidade comum e a proximidade física foram os fatores menos citados, presentes em apenas uma entrevista.

Neste mesmo contexto, as possíveis barreiras e suas relações, para o processo de compartilhamento, estão representadas na Figura 3. 


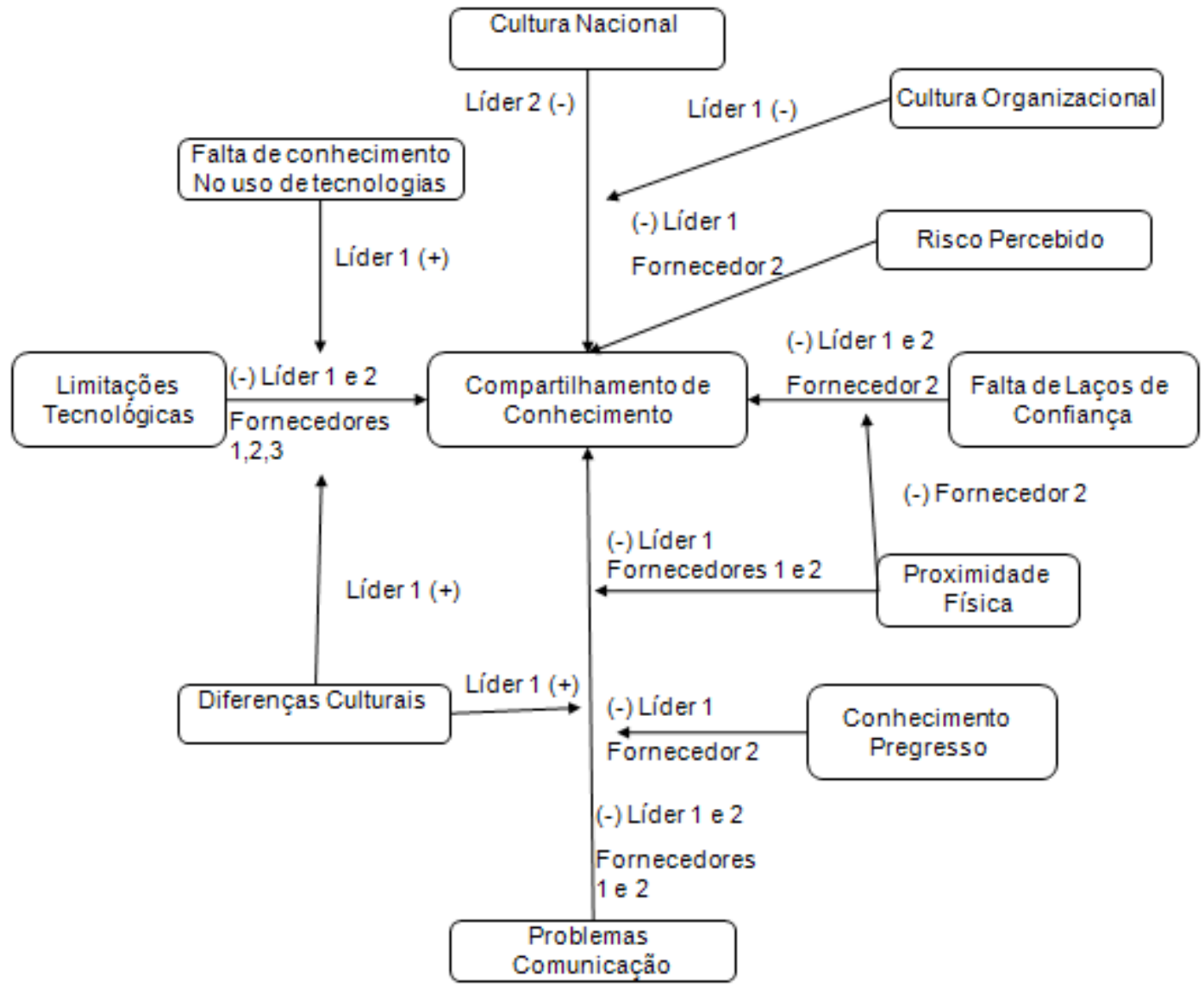

Figura 3 - Barreiras para o processo de compartilhamento de conhecimento em cadeias de suprimento

Fonte: os autores

Dentre as barreiras para o processo de compartilhamento de conhecimento, conforme representado na Figura 3, somente as limitações tecnológicas foram citadas por todos os entrevistados. A falta de laços de confiança, os problemas de comunicação, a falta de proximidade física, a falta de conhecimento pregresso e os riscos percebidos foram apontados como barreiras por dois entrevistados cada. Vale ressaltar o fator moderador do conhecimento pregresso sobre os problemas de comunicação, de acordo com dois entrevistados, e a percepção de um dos entrevistados sobre o fator moderador exercido pela proximidade física, reduzindo os efeitos da falta de confiança, e a moderação da falta de conhecimento no uso de tecnologia, potencializando o efeito das limitações tecnológicas. Na percepção de uma das empresas líderes de cadeia, as diferenças culturais potencializam os problemas de comunicação e as limitações tecnológicas.

\section{Conclusões}

Este artigo analisou como os elementos discutidos na literatura sobre compartilhamento de conhecimento são percebidos na visão de agentes da cadeia de suprimentos. Através da percepção dos entrevistados, pontos importantes como o efeito da cultura nacional, da confiança, da importância de conhecimentos pregressos, da necessidade da percepção de recompensas financeiras e econômicas, e do papel do gestor no compartilhamento de conhecimento foram identificados. Desta forma, as contribuições para os estudos relacionados ao compartilhamento de conhecimento e à gestão de cadeias de suprimentos foram elencadas, como a importância de redes de relacionamento e da habilidade de comunicação, a influência negativa das limitações tecnológicas associadas falta de conhecimento no uso de tecnologias e de diferenças culturais.

Em relação às implicações práticas e gerencias deste trabalho, para o contexto de uma cadeia de suprimentos, de acordo com os entrevistados, a manutenção da rede de relacionamentos e a habilidade de comunicação são pontos de atenção gerencial, visando estimular o processo de compartilhamento de conhecimento, conforme apontado por todos os entrevistados neste trabalho. $\mathrm{O}$ estabelecimento de um relacionamento recíproco e de 
laços de confiança, a intenção de compartilhar, o papel do gestor e da alta administração, por sua vez, também representam questões relevantes para este processo. $\mathrm{O}$ processo de compartilhamento pode ser beneficiado, ainda, com a redução dos fatores que podem constituir possíveis barreiras, como as limitações tecnológicas e os problemas de comunicação. A proximidade física e o conhecimento pregresso acerca do conhecimento compartilhado podem reduzir os efeitos negativos dos problemas de comunicação, enquanto a falta de conhecimento no uso de tecnologias e as diferenças culturas podem ampliar os efeitos negativos das limitações tecnológicas. Merece atenção, também, a percepção dos riscos acerca do compartilhamento, que também é percebida como uma barreira para o processo de compartilhamento, segundo os entrevistados neste trabalho.

Embora o reduzido número de entrevistados possa ser considerado uma limitação ao trabalho, o fato de que técnicas interpretativas buscam o significado e não a frequiência dos fenômenos que ocorrem no mundo social (VAN MAANEN, 1979) torna esta pesquisa adequada, devido ao seu caráter exploratório qualitativo. Desta forma, o complemento deste estudo com uma survey dedicada as cadeias de suprimentos emerge como pesquisa futura.

Uma questão que despertou interesse maior é o relacionamento entre o compartilhamento de conhecimento e os processos de qualidade envolvidos nas atividades das empresas pesquisadas. Neste sentido, pesquisas podem ser realizadas com o intuito de elencar o possível relacionamento entre os procedimentos formais relacionados com certificações e normatizações e a gestão do conhecimento, e, em particular, com o compartilhamento de conhecimento. Outro ponto é que as entrevistas demonstraram uma forte percepção dos agentes quanto à especificidade dos resultados em relação ao setor de atuação. Desta forma, outra possibilidade de pesquisa futura é a aplicação da mesma metodologia sobre outras estruturas de mercado, bem como a comparação de seus resultados.

Por fim, entende-se que a cultura nacional também confere unicidade aos resultados, o que acaba sendo outra limitação do estudo e torna interessante novos estudos baseadas em entrevistas que alcancem outros países e nacionalidades.

\section{Agradecimentos}

Os autores agradecem o suporte recebido do $\mathrm{CNPq}$ (Conselho Nacional de Desenvolvimento Científico e Tecnológico) e da CAPES (Coordenação de Aperfeiçoamento de Pessoal de Nível Superior).

\section{Referências}

ARGOTE, L.; INGRAM, P. Knowledge transfer: A basis for competitive advantage in firms. Organizational Behavior and Human Decision Processes, v. 82, n. 1, p. 150-169, 2000.

CHEN, I. J.; PAULRAJ, A. Towards a theory of supply chain management: the constucts and measurements. Journal of Operations Management, v. 22, n. 2, p. 119-150, 2004.

CHOU, S. Knowledge creation: Absorptive capacity, organizational mechanisms, and knowledge storage/ retrieval capabilities. Journal of Information Science, v. 31 , n. 6 , p. $453-465,2005$.

COOK, L. S.; HEISER, D. R.; SENGUPTA, K. The moderating effect of supply chain role on the relationship between supply chain practices and performance. International Journal of Physical Distribution \& Logistics Management, v. 41, n. 2, p. 104-134, 2011.

COOPER, M. C.; LAMBERT, D. M.; PAGH, J. D. Supply Chain Management: more than a new name for logistics. The International Journal of Logistics Management, v. 8, n. 1, p. 1-14, 1997.

DAS, T. K.; TENG, B. Trust, control, and risk in strategic alliances: An integrated framework. Organization Studies, v. 22, n. 2, p. 251-283, 2001.

DAVENPORT, T.; DE LONG, D.; BEERS, M. Successful Knowledge Management Projects. MIT Sloan Management Review, v. 39, n. 2, p. 43-57, 1998.

DONG, G.; CHAU, G. L.; GROSSMAN, M. Knowledge-sharing intention in Vietnamese organizations. VINE, v. 40, n. 3, p. 262-276, 2010.

EL HARBI, S.; ANDERSON, A. R.; AMAMOU, M. Knowledge sharing processes in Tunisian small ICT firms. Library Review, v. 60, n. 1, p. 24-36, 2011.

ESPALLARDO, M. H.; OREJUELA, A. R.; PÉREZ, M. S. Inter-organizational governance, learning and performance in supply chains. Supply Chain Management, v. 15, n. 2 , p. 101-114, 2010.

FLICK, U. An introduction to qualitative research. Thousand Oaks: Sage Publications, 2009.

FORD, D. P.; STAPLES, S. Are full and partial knowledge sharing the same? Journal of Knowledge Management, v. 14, n. 3, p. 394-409, 2010. 
FRIESL, M.; SACKMANN, S.; KREMSER, S. Knowledge sharing in new organizational entities: The impact of hierarchy, organizational context, micro-politics and suspicion. Cross Cultural Management, v. 18, n. 1, p. 71-86, 2011.

FUNDAÇÃO DE ECONOMIA E ESTATÍSTICA. Apresentação: PIB-RS. Porto Alegre. Disponível em: $<$ http://www.fee.tche.br/sitefee/pt/content/estatisticas/ pg_pib.php>. Acesso em: 29 nov. 2011.

GIBBS, G. Analyzing qualitative data. Thousand Oaks: SAGE, 2009.

HAKANSSON, H.; PERSSON, G. Supply Chain Management: The Logic of Supply Chains and Networks. International Journal of Logistics Management, v. 15, n. 1, p. 11-26, 2004.

HOOFF, B.V. D.; DE RIDDER, J. Knowledge sharing in context: the influence of organizational commitment, communication climate and CMC use on knowledge sharing. Journal of Knowledge Management, v. 8, n. 6, p. 117-130, 2004.

KARKOULIAN, S.; AL HARAKE, N.; MESSARRA, L. C. Correlates of Organizational Commitment and Knowledge Sharing via Emotional Intelligence: An Empirical Investigation. The Business Review, Cambridge, v. 15, n. 1, p. 89-96, 2010.

KHAN, I. A. Knowledge Groups: A Model for Creating Synergy Across the Public Sector. Public Organization Review, v. 10, n. 2, p. 139-152, 2010.

LAURING, J.; SELMER, J. Multicultural organizations: common language, knowledge sharing and performance. Personnel Review, v. 40, n. 3, p. 324-343, 2011.

LI, W. Virtual knowledge sharing in a cross-cultural context. Journal of Knowledge Management, v. 14, n. 1, p. 38-50, 2010.

LILLEOERE, A.; HANSEN, E. H. Knowledge-sharing enablers and barriers in pharmaceutical research and development. Journal of Knowledge Management, v. 15, n. 1, p. 53-70, 2011.

MALhOTRA, A.; GOSAIN, S.; EL SAWY, O. A. Absorptive capacity configurations in supply chains: Gearing for partner-enabled market knowledge creation. MIS Quarterly, v. 29, n. 1, p. 145-187, 2005.

MALHOTRA, N. K. Pesquisa de marketing: uma orientação aplicada. 3. ed. Porto Alegre: Bookman, 2001.
MANJIT, S. S.; JAIN, K.; AHMAD, I. U. K. Knowledge sharing among public sector employees: evidence from Malaysia. The International Journal of Public Sector Management, v. 24, n. 3, p. 206-226, 2011.

MEHRJERDI, Y. Z. The collaborative supply chain. Assembly Automation, v. 29, n. 2, p. 127-136, 2009.

NONAKA, I.; TAKEUCHI, H. The Knowledge-creating company: How Japanese companies create the dynamics of innovation. Oxford University Press: New York, 1995.

RIEGE, A. Three-dozen knowledge-sharing barriers managers must consider. Journal of Knowledge Management, v. 9, n. 3, p. 18-35, 2005.

SIAKAS, K. V.; GEORGIADOU, E.; BALSTRUP, B. Cultural impacts on knowledge sharing: empirical data from EU project collaboration. VINE, v. 40, n. 3/4, p. 376-389, 2010.

SUPPIAH, V.; MANJIT, S.S. Organisational culture's influence on tacit knowledge-sharing behaviour. Journal of Knowledge Management, v. 15, n. 3, p. 462-477, 2011.

SWIFT, M.; BALKIN, D. B.; MATUSIK, S. F. Goal orientations and the motivation to share knowledge. Journal of Knowledge Management, v. 14, n. 3, p. 378-393, 2010.

TENG, J. T. C.; SONG, S. An exploratory examination of knowledge-sharing behaviors: solicited and voluntary. Journal of Knowledge Management, v. 15, n. 1, p. 104-117, 2011.

THOMAS, R. W.; ESPER, T. L.; STANK, T. P. Testing the Negative Effects of Time Pressure in Retail Supply Chain Relationships. Journal of Retailing, v. 86, n. 4, p. 368-382, 2010.

TOHIDINIA, Z.; MOSAKHANI, M. Knowledge sharing behaviour and its predictors. Industrial Management + Data Systems, v. 110, n. 4, p. 611-631, 2010.

VAN MAANEN, J. Reclaiming Qualitative Methods for Organizational Research: A Preface. Administrative Science Quarterly, v. 24, n. 4, p. 520-526, 1979.

VANPOUCKE, E.; BOYER, K. K.; VEREECKE, A. Supply chain information flow strategies: an empirical taxonomy. International Journal of Operations \& Production Management, v. 29, n. 12, p. 1213-1241, 2009.

VELMURUGAN, M.; KOGILAH, N.; DEVINAGA, R. Knowledge Sharing in Virtual Teams in Malaysia: 
Its Benefits and Barriers. Journal of Information \& Knowledge Management, v. 9, n. 2, p. 145-159, 2010.

WISNER, J. D. Supply Chain Management and its impact on purchasing. Journal of Supply Chain Management, v. 36, n. 4, p. 33-42, 2000.

Recebido em 26/04/2012.

Aceito em 06/08/2012. 